This item was submitted to Loughborough's Research Repository by the author.

Items in Figshare are protected by copyright, with all rights reserved, unless otherwise indicated.

\title{
Reply to comment by Lu et al. on "An efficient and stable hydrodynamic model with novel source term discretization schemes for overland flow and flood simulations"
}

\section{PLEASE CITE THE PUBLISHED VERSION}

https://doi.org/10.1002/2017WR021696

\section{PUBLISHER}

(c) American Geophysical Union (AGU)

\section{VERSION}

VoR (Version of Record)

\section{PUBLISHER STATEMENT}

This work is made available according to the conditions of the Creative Commons Attribution-NonCommercialNoDerivatives 4.0 International (CC BY-NC-ND 4.0) licence. Full details of this licence are available at: https://creativecommons.org/licenses/by-nc-nd/4.0/

\section{LICENCE}

CC BY-NC-ND 4.0

\section{REPOSITORY RECORD}

Xia, Xilin, Qiuhua Liang, Xiaodong Ming, and Jingming Hou. 2019. "Reply to Comment by Lu Et Al. On "an Efficient and Stable Hydrodynamic Model with Novel Source Term Discretization Schemes for Overland Flow and Flood Simulations"”. figshare. https://hdl.handle.net/2134/34202. 


\section{Water Resources Research}

\section{REPLY \\ 10.1002/2017WR021696}

This article is a reply to Lu et al. [2018], https://doi.org/10.1002/2017WR021563.

\section{Key Points:}

- The alternative method by Lu et al. (2017) is effective, but not necessarily easier to implement or more efficient - Small numerical wiggles do not affect the overall solution accuracy of the surface reconstruction method (SRM) - Hydrostatic reconstruction may perform equally well if highly refined grids are used

Correspondence to:

Q. Liang,

qiuhua.liang@ncl.ac.uk

Citation:

Xia, X., Liang, Q., Ming, X., \& Hou, J. (2018). Reply to comment by Lu et al. on "An efficient and stable hydrodynamic model with novel source term discretization schemes for overland flow and flood simulations". Water Resources Research, 54. https:// doi.org/10.1002/2017WR021696

Received 10 AUG 2017 Accepted 19 DEC 2017 Accepted article online 28 DEC 2017

(c) 2017. American Geophysical Union. All Rights Reserved.

\section{Reply to Comment by Lu et al. on "An Efficient and Stable Hydrodynamic Model With Novel Source Term Discretization Schemes for Overland Flow and Flood Simulations"}

\author{
Xilin Xia' ${ }^{1}$, Qiuhua Liang ${ }^{1}$ iD, Xiaodong Ming ${ }^{1}$ (D), and Jingming Hou² \\ ${ }^{1}$ School of Engineering, Newcastle University, Newcastle Upon Tyne, UK, ${ }^{2}$ School of Water Resources and Hydro-Power \\ Engineering, Xi'an University of Technology, Xi'an, China
}

\begin{abstract}
This document addresses the comments raised by Lu et al. (2017). Lu et al. (2017) proposed an alternative numerical treatment for implementing the fully implicit friction discretization in Xia et al. (2017). The method by Lu et al. (2017) is also effective, but not necessarily easier to implement or more efficient. The numerical wiggles observed by Lu et al. (2017) do not affect the overall solution accuracy of the surface reconstruction method (SRM). SRM introduces an antidiffusion effect, which may also lead to more accurate numerical predictions than hydrostatic reconstruction (HR) but may be the cause of the numerical wiggles. As suggested by Lu et al. (2017), HR may perform equally well if fine enough grids are used, which has been investigated and recognized in the literature. However, the use of refined meshes in simulations will inevitably increase computational cost and the grid sizes as suggested are too small for real-world applications.
\end{abstract}

We thank Lu, Mao, and Dong (Lu et al., 2017, hereafter referred to as LMD) for their interest in our work and providing detailed comments on the Surface Reconstruction Method (SRM) and the implicit friction discretization method we reported (Xia et al., 2017, hereafter referred to as X17). We largely concur with their suggestion that more studies are needed for solving the shallow water equations and hope our work would stimulate more research in this field.

LMD's comments may be summarized into three points: (1) LMD provides an easier to implement and more efficient numerical treatment as an alternative to the introduction of auxiliary variables for implementing the fully implicit friction discretization in X17; (2) SRM may create numerical wiggles in certain test cases; and (3) the original hydrostatic reconstruction (HR) method works equally well if sufficiently fine grids are used. In the remaining part of this document, we will provide detailed point-to-point response to these comments and finally point out a few minor mistakes in LMD's original comment document.

C1: As agreed by LMD, the possible extreme value caused by $h^{-7 / 3}$ in the friction terms when the water depth is small may generate machine error and must be avoided. Numerical treatment is indeed essential. The introduction of auxiliary variables as proposed in X17 provides a simple but effective approach to avoid the possible numerical issue. LMD provides an alternative method to deal with the same numerical issue. It is true that the method in LMD does not require additional local variables to store the auxiliary variables. But such a difference is too insignificant to affect the overall computational efficiency. In addition, equation (5) introduced in LMD for calculating the Jacobian matrix is slightly more complex than that in X17, and requires careful ordering of the arithmetic calculations. Therefore, the method in LMD is not necessarily easier to implement. Saying this, the alternative method proposed by LMD is also proved to be effective.

C2: LMD has observed small numerical wiggles in a test case involving an oscillating water body moving in a parabolic bowl. This has pointed out a direction to further improve SRM and develop improved numerical schemes for solving the shallow water equations. Identifying the cause of these wiggles and designing methods to remove them should, consequently, be a clear priority for future research. However, at this moment, we would restrict ourselves to asking: (1) will these small numerical wiggles affect the overall solution accuracy? and (2) will the numerical inaccuracy as introduced by these wiggles grow with time and lead to divergent solutions? To answer these questions, we present the numerical results for the same test case obtained on three different grids, i.e., 1,10 , and $100 \mathrm{~m}$. The $L_{1}, L_{2}$, and $L_{\infty}$ errors calculated for the numerical solutions at $t=5 \mathrm{~T}(\mathrm{~T}=1,346.4 \mathrm{~s}$ is the oscillation period of the flow) are listed in Table 1. It is evident that both SRM and HR compute results converging to the analytical solution as the grids being refined. 


\section{QAGU Water Resources Research}

Table 1

Numerical Errors Calculated for Different Simulations at $\mathrm{t}=5 \mathrm{~T}$

\begin{tabular}{llll}
\hline Simulations & \multicolumn{1}{c}{$L_{1}$} & \multicolumn{1}{c}{$L_{2}$} & \multicolumn{1}{c}{$L_{\infty}$} \\
\hline SRM $100 \mathrm{~m}$ & $1.629 \times 10^{-1}$ & $5.956 \times 10^{-2}$ & $7.053 \times 10^{-1}$ \\
HR $100 \mathrm{~m}$ & $1.114 \times 10^{0}$ & $2.476 \times 10^{0}$ & $3.446 \times 10^{0}$ \\
SRM $10 \mathrm{~m}$ & $1.747 \times 10^{-2}$ & $9.032 \times 10^{-4}$ & $1.634 \times 10^{-1}$ \\
HR $10 \mathrm{~m}$ & $5.335 \times 10^{-2}$ & $6.090 \times 10^{-3}$ & $1.930 \times 10^{-1}$ \\
SRM 1 m & $2.842 \times 10^{-3}$ & $2.444 \times 10^{-5}$ & $3.431 \times 10^{-2}$ \\
HR 1 m & $4.078 \times 10^{-3}$ & $3.659 \times 10^{-5}$ & $1.615 \times 10^{-2}$ \\
\hline
\end{tabular}

For all of the simulations, the numerical errors predicted by SRM are smaller than those resulting from HR except for the slightly bigger SRM $L_{\infty}$ error for the $1 \mathrm{~m}$ simulation. Particularly, the SRM $L_{1}$ and $L_{2}$ errors, which reflect the overall solution accuracy, are all much smaller than the corresponding HR errors on coarser grids (10 and $100 \mathrm{~m}$ ). Taking a closer look at the $100 \mathrm{~m}$ simulations, the SRM and HR results at $t=1 \mathrm{~T}$ are similar but the water level profile predicted by HR has clearly departed from the analytical solution at $t=5 \mathrm{~T}$ due to the energy dissipation introduced by HR's large numerical diffusion. However, the numerical wiggles predicted by SRM do not grow with time. Therefore, we may conclude that SRM works well for this test case and it clearly outperforms HR on coarser grid simulations. Also from the numerical results as presented in $\mathrm{X} 17$, the numerical wiggles do not cause any noticeable concerns for real-world applications.

From the theoretical point of view, it will be interesting to know what causes the numerical wiggles. LMD reckoned that the cause of the numerical wiggles may be because SRM "may miss representing the gravitational effects." This could be a reason but we need further elaboration to prove it. Herein, we will share our own thoughts. From the results as shown in Figure 1, the additional reconstruction "error" introduced by SRM somehow suppresses the excessive numerical diffusion introduced by HR. We conjecture that the numerical wiggles may be caused by the SRM's antidiffusion effect, which is not necessarily a disadvantage and evidently gives more accurate results than HR on coarser grids in the test case as shown. More research is needed to theoretically investigate this issue.

C3: LMD has further tested the HR method by performing simulations with varying grid resolutions, using the test case of steady uniform flow on slopes. Their results indicate that the numerical inaccuracy caused by HR may be resolved by using highly refined grids. This is not a surprising conclusion and has already been investigated and recognized (e.g., Delestre et al., 2012). However, the use of refined meshes will inevitably increase computational cost and the spatial resolution as recommended by LMD, i.e., 0.1 and $0.001 \mathrm{~m}$, is unrealistically small for real-world overland flow simulations.

Finally, we would like to point out a few minor mistakes in LMD's original document:

The HR of the discharge in Hou et al. (2015) is indeed mathematically the same as the one introduced in Audusse et al. (2004).

The expression of the Sampson's analytical solution is not correct and the correct formula is

$$
\eta(x, t)=h_{0}-\frac{a^{2} B^{2} s^{2}}{8 g^{2} h_{0}} \cos 2 s t-\frac{B^{2}}{4 g}-\frac{B s x}{g} \cos s t
$$

$\frac{t_{f}}{t_{H D}}$ should be changed to $\frac{t_{H D}}{t_{f}}$; otherwise the solution would reach the steady states in approximately a single step rather than many steps as indicated by LMD.

Table 1, row 8, column 2: The expressions should be $\left(Q_{x}\right)_{L}=u_{i} h_{L},\left(Q_{x}\right)_{R}=u_{i+1} h_{R}$.
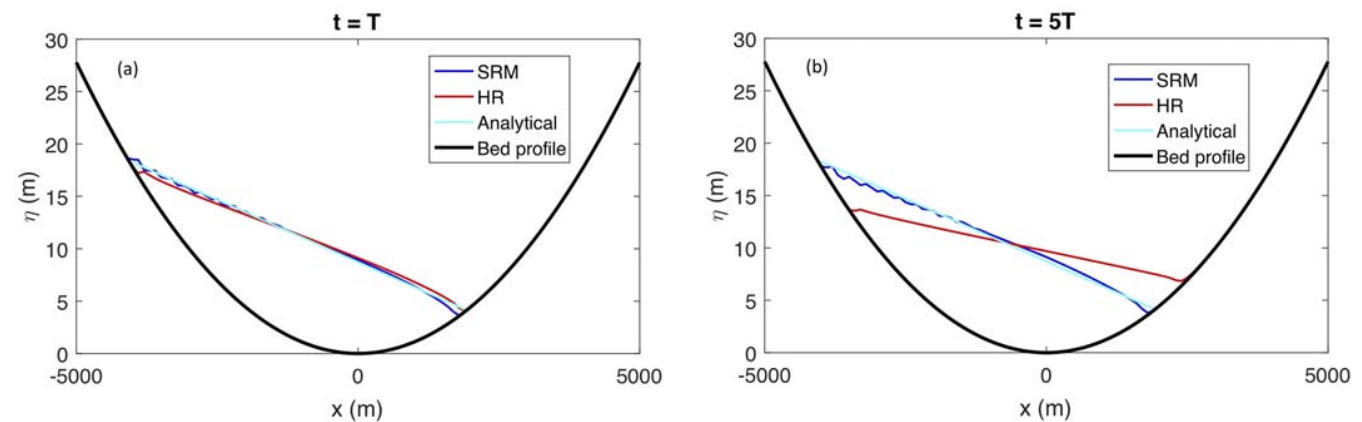

Figure 1. Results obtained on $100 \mathrm{~m}$ grid: (a) $t=\mathrm{T}$ and (b) $t=5 \mathrm{~T}$. 


\section{QAGU Water Resources Research}

Acknowledgment

Please see Xia et al. (2017) for the data

referred to in this paper.

\section{References}

Audusse, E., Bouchut, F., Bristeau, M.-O., Klein, R., \& Perthame, B. (2004). A fast and stable well-balanced scheme with hydrostatic reconstruction for shallow water flows. SIAM Journal on Scientific Computing, 25(6), 2050-2065. https://doi.org/10.1137/S1064827503431090

Delestre, O., Cordier, S., Darboux, F., \& James, F. (2012). A limitation of the hydrostatic reconstruction technique for shallow water equations. Comptes Rendus Mathématique, 350(13-14), 677-681. https://doi.org/10.1016/j.crma.2012.08.004

Hou, J., Liang, Q., Zhang, H., \& Hinkelmann, R. (2015). An efficient unstructured MUSCL scheme for solving the 2D shallow water equations. Environmental Modelling \& Software, 66, 131-152. https://doi.org/10.1016/j.envsoft.2014.12.007

Lu, X., Mao, B., \& Dong, B. (2017). Comment on "An efficient and stable hydrodynamic model with novel source term discretization schemes for overland flow and flood simulations. Water Resources Research. https://doi.org/10.1002/2017WR021563

Xia, X., Liang, Q., Ming, X., \& Hou, J. (2017). An efficient and stable hydrodynamic model with novel source term discretization schemes for overland flow and flood simulations. Water Resources Research, 53, 3730-3759. https://doi.org/10.1002/2016WR020055 